\title{
High tumor budding stratifies breast cancer with metastatic properties
}

\author{
Bodour Salhia $^{1}$ - Mafalda Trippel ${ }^{2} \cdot$ Katrin $_{\text {Pfaltz }^{2}} \cdot$ Nikola Cihoric $^{3,4}$. \\ André Grogg ${ }^{2}$ - Claudia Lädrach ${ }^{2}$ Inti Zlobec ${ }^{5}$ Coya Tapia ${ }^{2,4}$
}

Received: 14 December 2014/ Accepted: 4 March 2015/Published online: 17 March 2015

(C) The Author(s) 2015. This article is published with open access at Springerlink.com

\begin{abstract}
Tumor budding refers to single or small cluster of tumor cells detached from the main tumor mass. In colon cancer high tumor budding is associated with positive lymph nodes and worse prognosis. Therefore, we investigated the value of tumor budding as a predictive feature of lymph node status in breast cancer (BC). Whole tissue sections from 148 surgical resection specimens (SRS) and 99 matched preoperative core biopsies (CB) with invasive BC of no special type were analyzed on one slide stained with pan-cytokeratin. In SRS, the total number of intratumoral (ITB) and peripheral tumor buds (PTB) in ten high-power fields (HPF) were counted. A bud was defined as a single tumor cell or a cluster of up to five tumor cells. High tumor budding equated to scores averaging $>4$ tumor buds across 10HPFs. In CB high tumor budding was defined as $\geq 10$ buds/HPF. The results were correlated with pathological parameters. In SRS high PTB stratified $\mathrm{BC}$ with lymph node metastases $(p \leq 0.03)$ and lymphatic invasion $(p \leq 0.015)$. In CB high tumor budding was significantly $(p=0.0063)$ associated with venous
\end{abstract}

Bodour Salhia

bsalhia@tgen.org

Coya Tapia

coya.tapia@hotmail.com

1 Integrated Cancer Genomics Division, Translational Genomics Research Institute, 445 N. Fifth Street, Phoenix, AZ 85004, USA

2 Division of Clinical Pathology, Institute of Pathology, University of Bern, Murtenstrasse 31, 3010 Bern, Switzerland

3 Department of Radiation Oncology, Bern University Hospital, and University of Bern, Bern, Switzerland

4 Breast Center, University Hospital Bern, Bern, Switzerland

5 Translational Research Unit (TRU), Institute of Pathology, University of Bern, Bern, Switzerland invasion. Pathologists are able, based on morphology, to categorize $\mathrm{BC}$ into a high and low risk groups based in part on lymph node status. This risk assessment can be easily performed during routine diagnostics and it is time and cost effective. These results suggest that high PTB is associated with loco-regional metastasis, highlighting the possibility that this tumor feature may help in therapeutic decision-making.

Keywords Breast cancer - No special type - Tumor budding $\cdot$ Vessel invasion $\cdot$ Metastasis

\section{Background}

Breast cancer (BC) is considerably heterogeneous both molecularly and histologically. It is presented as a collection of distinct disease subtypes that differ in disease progression, treatment response, and disease-free survival [1-5]. A variety of clinical pathological and genetic factors are routinely used to assess prognosis and determine the most appropriate therapeutic regimen for patients with $\mathrm{BC}[6,7]$. These include patient age, axillary lymph node status, tumor size, histological grade, lymphatic invasion, hormone receptor status, HER2 status, and evaluation of tumor margins. More recently, molecular tests such as Oncotype DX ${ }^{\circledR}$ and MammaPrint ${ }^{\circledR}$ are being utilized to assess likelihood of treatment response and/or recurrence. However, additional prognosticators are needed to enhance personalized treatment and especially to overcome over and under treatment of patients.

Despite the substantial molecular and morphological differences, all invasive BC subtypes likely share a pathway for invasion, evidenced from morphological changes that occur in the transition to all invasive carcinomas. Normal epithelia, including pre-invasive breast cancers (carcinoma in situ), are physically separated from 
surrounding stroma and vascular structures by a basement membrane and a layer of myoepithelial cells [3]. Disruption of this barrier is a pre-requisite for invasion in all breast cancers. In addition, the invasive front of breast cancer also includes infiltrates of diverse immune cells including macrophages, neutrophils, and mast cells, and displays angiogenesis, and desmoplasia with deposition of extracellular matrix (ECM), particularly collagen I [8].

In colorectal carcinomas (CRC), morphological studies of the invasive front have revealed the emergence of tumor budding, which reflect detachment of tumor cells into single cells or clusters of up to five cells [9]. Tumor budding is diagnosed at high magnification and should not be mistaken with the tumor border configuration, which is more easily discernable at low magnifications. Biologically, the role of tumor budding includes a mechanism for invasive cells to migrate through peri-tumoral connective tissue, evade the host's defensive mechanisms and invade lymphatic and blood vessels with the consequence of local and distant metastases [9].

Over the last few years, numerous publications have shown the importance of tumor budding as an independent predictor of lymph node positivity, local and distant relapse, lymphatic invasion, and poor prognosis among patients with CRC of all pathological stages [9]. However, the definite implementation of tumor budding into clinical practice is currently limited by the lack of an internationally standardized scoring system. Whether tumor budding can be considered a histomorphologic features in $\mathrm{BC}$ remains to be elucidated. The purpose of the current study was to evaluate the potential clinical value of tumor budding in breast cancer of no special type (NST) as a predictor of lymph node metastasis.

\section{Materials and methods}

\section{Patient samples}

We searched the database at the Institute of Pathology, University of Bern, Switzerland for patients diagnosed with primary BC between January 2005 and December 2011. We identified 356 patients with suitable formalin fixed and paraffin-embedded (FFPE) samples from female patients with therapy naïve, primary, unilateral $\mathrm{BC}$ of more than $5 \mathrm{~mm}$ in size to construct a next-generation tissue microarray (ngTMA). From these patients, we selected 148 individual surgical resection specimens (SRS) and 99 matching preoperative core biopsies (CB) from patients with invasive BC of no special type (NST) and known lymph node status for tumor budding analysis. The TNM classification was taken from the pathology reports, and the clinical data were extracted from the database of the breast center of the University Hospital Bern, Switzerland. The median age of selected patients at the time of diagnosis was 61 years (range 32-91 years). The clinico-pathological features for the sample cohort are summarized in Table 1, and the study design is presented in Fig. 1.

All BCs were reviewed and the histological subtype and tumor grade were assigned according to the WHO classification 2012. The study was approved by the ethical committee of the University of Bern (Registration 200/2014) and was performed according to the REMARK guidelines [10]. Long-term follow-up data were not available for this cohort.

\section{Next-generation tissue microarray (ngTMA)}

For the assessment of the prognostic factors, a ngTMA was constructed including 356 selected BC samples as previously described [11]. In brief, whole tissue sections of the tumors were scanned and uploaded to a digital platform to make annotations for the punching process. The tumor image on the screen allowed an optimal overview of the sample for digital annotations of the regions of intersest, including areas from the tumor center and periphery. The fully automated arraying process synchronized the annotations made on the scanned slide with the image of the donor paraffin block to precisely punch the area of interest. For the majority $(97.8 \%)$ of BC samples, six punches of $0.6 \mathrm{~mm}$ were integrated into a new recipient block representing the ngTMA.

\section{Immunohistochemistry (IHC) and fluorescence in situ hybridization (FISH)}

Prior to ngTMA construction, $3 \mu \mathrm{m}$ whole tissue sections from one selected FFPE tissue block of the 148 NST BCs were cut and incubated with an anti-cytokeratin antibody for better visualization of tumor buds for evaluation. Estrogen (ER) and progesterone (PgR) receptor, Her2 and MIB1 were performed on the ngTMA. The antibodies and the specific conditions used are listed in Table 2.

For ER, PgR, and Her2, the ASCO/CAP guidelines were applied to define positive hormone receptor and Her2 status, respectively $[12,13]$. In $\mathrm{BC}$ with equivocal immunohistochemistry, fluorescence in situ hybridization (FISH) was performed using a dual-color probe (Abbott Molecular Abbott Park, Illinois, USA).

The molecular subtypes were defined as follows: Luminal A: estrogen (ER) and/or progesterone receptor (PgR) positive, Her2 negative, low proliferative index $(\leq 15 \%)$; Luminal B: hormone receptor positive, Her2 positive/or high proliferative index ( $>15 \%)$; Her2 subtype: Her2 
Table 1 Patient characteristics $(n=148)$

\begin{tabular}{|c|c|c|}
\hline \multicolumn{2}{|l|}{ Feature } & \multirow{2}{*}{$\frac{\text { Frequency N }(\%)}{61(32,91)}$} \\
\hline Age (years) & Median (min, max) & \\
\hline Tumor size $(\mathrm{cm})$ & Median (min, max) & $2.0(0.6-8.0)$ \\
\hline \multirow[t]{2}{*}{ ER } & Positive & $125(84.5)$ \\
\hline & Negative & $23(15.5)$ \\
\hline \multirow[t]{2}{*}{$\mathrm{PgR}$} & Positive & $104(70.3)$ \\
\hline & Negative & $44(29.7)$ \\
\hline \multirow[t]{2}{*}{ Her 2} & Positive & $19(12.8)$ \\
\hline & Negative & $129(87.2)$ \\
\hline \multirow[t]{3}{*}{ MIB1 } & High $(>15 \%)$ & $40(27.0)$ \\
\hline & Low $(\leq 15 \%)$ & $107(72.3)$ \\
\hline & No data & $1(0.7)$ \\
\hline \multirow[t]{3}{*}{ Grade } & G1 & $18(12.2)$ \\
\hline & $\mathrm{G} 2$ & 69 (46.6) \\
\hline & G3 & $61(41.2)$ \\
\hline \multirow[t]{4}{*}{ pT } & $\mathrm{T} 1$ & $75(50.7)$ \\
\hline & $\mathrm{T} 2$ & $66(44.6)$ \\
\hline & $\mathrm{T} 3$ & $3(2.0)$ \\
\hline & $\mathrm{T} 4$ & $4(2.7)$ \\
\hline \multirow[t]{2}{*}{$\mathrm{pN}$} & pNO & $68(45.9)$ \\
\hline & $>\mathrm{pNO}$ & $80(54.1)$ \\
\hline \multirow[t]{4}{*}{ Molecular subtypes } & Luminal A & $92(62.2)$ \\
\hline & Luminal B & $33(22.3)$ \\
\hline & Her2 & $3(2.0)$ \\
\hline & Triple negative & $20(13.5)$ \\
\hline \multirow[t]{3}{*}{ Lymphatic invasion } & No & $89(60.1)$ \\
\hline & Yes & $58(39.5)$ \\
\hline & No data & $1(0.7)$ \\
\hline \multirow[t]{3}{*}{ Venous invasion } & No & $135(91.2)$ \\
\hline & Yes & $12(8.1)$ \\
\hline & No data & $1(0.7)$ \\
\hline \multirow[t]{3}{*}{ Perineural invasion } & No & $128(86.5)$ \\
\hline & Yes & $19(12.8)$ \\
\hline & No data & $1(0.7)$ \\
\hline \multirow[t]{3}{*}{ Hormone therapy } & No & $55(37.2)$ \\
\hline & Yes & $38(25.7)$ \\
\hline & Unknown & $55(37.1)$ \\
\hline \multirow[t]{3}{*}{ Chemotherapy } & No & $58(39.2)$ \\
\hline & Yes & $34(23.0)$ \\
\hline & Unknown & $56(37.8)$ \\
\hline \multirow[t]{3}{*}{ Anti-Her2 therapy } & No & $81(54.7)$ \\
\hline & Yes & $5(3.4)$ \\
\hline & Unknown & $62(41.9)$ \\
\hline \multirow[t]{3}{*}{ Radiotherapy } & No & $32(21.6)$ \\
\hline & Yes & 106 (71.6) \\
\hline & Unknown & $10(6.8)$ \\
\hline
\end{tabular}

positive and hormone receptor negative, any proliferative index; triple negative: Her2 negative, hormone receptor negative, any proliferative index.

\section{Scoring of tumor buds}

Tumor buds were defined as isolated single cancer cells or microscopic cell clusters composed of 1-5 cells. The number of buds was counted on whole tissue sections of surgical resection specimens (SRS; $n=148$ ) and matched preoperative core biopsies $(\mathrm{CB} ; n=99)$ stained with a pan-cytokeratin antibody (AE1/AE3) for better visualization. Areas with the highest concentration of buds ("hotspots") were selected for scoring. BC samples were selected from the cohort of the ngTMA as described earlier.

In SRS, tumor buds at the invasive front or within the tumor center were scored separately. Invasive tumor front buds are referred to as peripheral tumor buds (PTB) and were scored within $\approx 1.1 \mathrm{~mm}(2 \times 1$ high-power field (HPF) @ $0.55 \mathrm{~mm}^{2}$ ) on either direction of the tumor interface. Tumor buds within the center are referred to as intratumoral buds (ITB) (Fig. 2). We used pre-defined criteria for the assessment of tumor buds in accordance with recent publications describing scoring of tumor buds in colon cancer SRS [14] and cut-off criteria in BC [15]. For SRS, 2 pathologists (CT, MT) scored 10 HPF independently and blindly without knowledge of tumor characteristics (e.g., nodal status) and each other's results. According to a recent report [15], tumors were considered to have high tumor budding if the average number of tumor buds in $10 \mathrm{HPF}>4$. In contrast, tumors were considered to have low tumor budding if the average number of buds in $10 \mathrm{HPF}$ was $\leq 4$. Examples of high tumor budding are seen in Fig. 3 and examples of low tumor budding are given in Fig. 4.

Since CBs are not easily comparable to SRSs, we performed a receiver operating characteristic (ROC) curve analysis to find the appropriate cut-off for high and low tumor budding. In addition, we scored only $1 \mathrm{HPF}$ with the highest density of tumor buds as previously reported for preoperative colon biopsies [16]. Furthermore, ITB and PTB cannot be distinguished in CB because they are randomly sampled. One pathologist (CT) scored tumor budding in all CBs. In all SRSs and CBs tumor buds were counted at $\times 400$ magnification $\left(0.55 \mathrm{~mm}^{2}\right)$.

\section{Statistics}

The Chi Square test was used to calculate significant differences between categorical variables. Pearson's correlation coefficient was used to determine the strength of the linear relationship between budding in CB and SRS (ITB and PTB). A $p$ value $<0.05$ was considered statistically significant. Interrater reliability was calculated with Cohen's Kappa test with the following interpretations for the kappa value $0-0.2=$ poor; $0.21-0.4=$ fair; 
Fig. 1 Flow chart study

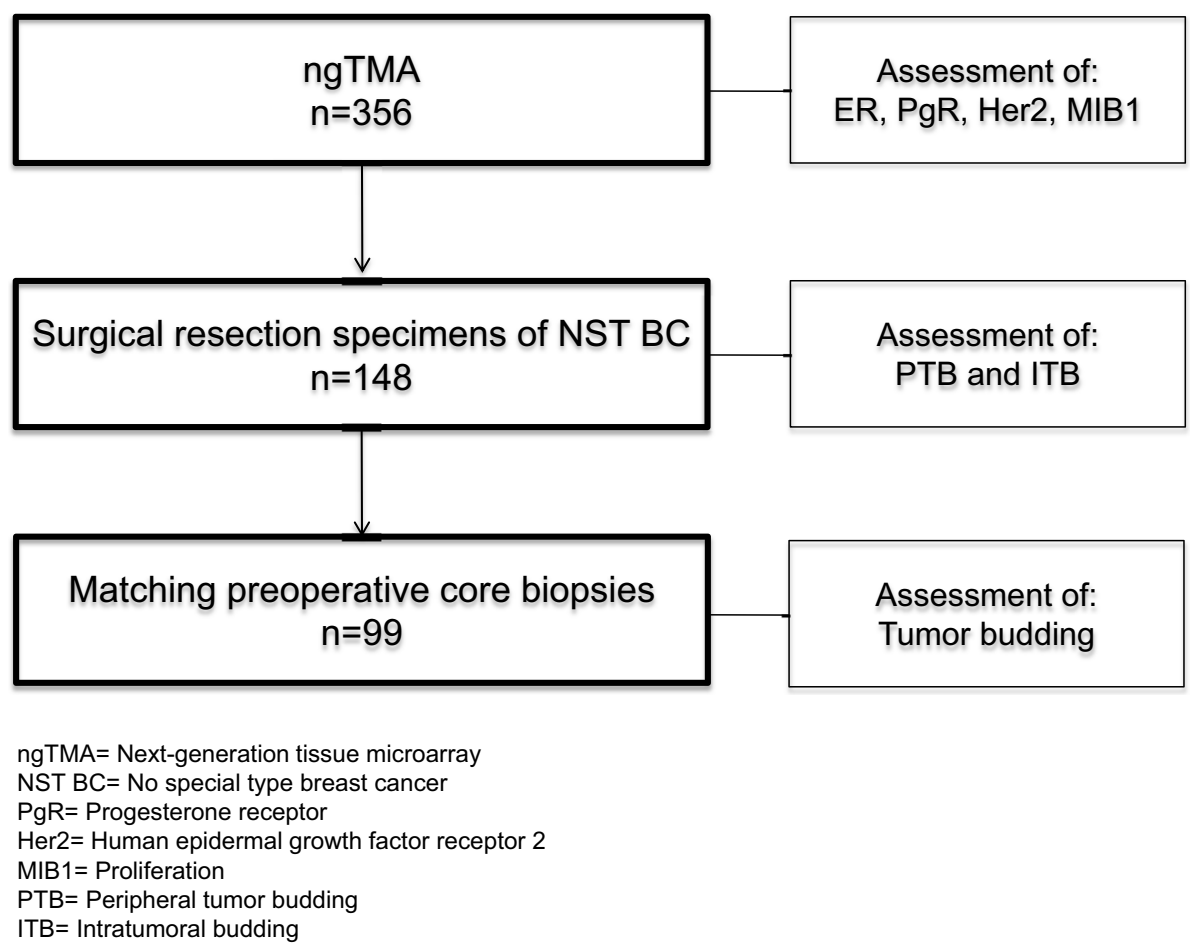

Table 2 Specifications of immunohistochemistry and antibodies used

\begin{tabular}{lllll}
\hline Antibody & Clone & Cat\#/vendor & Dilution & Retrival \\
\hline Estrogen receptor & EP1 & M3643/Dako & $1: 50$ & Tris pH 8.4 40 min 95 ${ }^{\circ} \mathrm{C}$ \\
Progesterone receptor & 16 SAN27 & NCL-L-PGR-AB/Novocastra & $1: 200$ & Tris pH 8.440 min $95{ }^{\circ} \mathrm{C}$ \\
Her2 & Poly & K5207/Dako & Kit & citrat pH6 20 min $100{ }^{\circ} \mathrm{C}$ \\
MIB1 & Mib1 & M7240/Dako & $1: 200$ & Tris pH 8.4 30 min 95 ${ }^{\circ} \mathrm{C}$ \\
Cytokeratin & AE1/AE3 & M351501-2/Dako & $1: 300$ & Proteinase K 5 min 37 ${ }^{\circ} \mathrm{C}$ \\
\hline
\end{tabular}

$0.41-0.6=$ moderate; $0.61-0.8=$ substantial; $>0.8=$ excellent [17]. To calculate a cut-off for high and low tumor budding in $\mathrm{CB}$, receiver operating characteristic (ROC) curve analysis was performed. Analyses were carried out using SPSS 21 (IBM, Armonk, US) and SAS (V9.4, The SAS Institute, Cary, NC).

\section{Results}

A total of 148 surgical resection specimens (SRS) and 99 matching preoperative core biopsies (CB) with invasive NST BC were evaluated. ER, PgR, Her2, and MIB1 proliferative index were obtained from ngTMA evaluation and are summarized in Table 1. In two patients, Her2 status $(1.4 \%)$ and in three patients MIB1 $(2.0 \%)$ results were taken from the pathological reports due to insufficient material present for evaluation on the ngTMA.

The concordance of the two pathologist regarding scoring of high and low tumor budding was $k=0.526$ for PTB and $k=0.533$ for ITB.

\section{Correlation of tumor budding with clinical- pathological characteristics}

Significant associations with PTB in surgical resection specimens

The individual scores of pathologists showed a significant correlation of high PTB with positive lymph node status ( $p=0.003$ and 0.03) and lymphatic invasion (LVI) ( $p=0.015$ and 0.013 ), respectively. The only significant result when combining the scoring of both pathologists was the association of high PTB with lymphatic invasion $(p=0.029)$. In addition, analysis of only ER-positive and low proliferative $\mathrm{BCs}$, respectively, showed the following results: Pathologist 1 showed a significant correlation with lymph node metastasis and high PTB when analyzing only ER-positive BCs $(p=0.007)$ and in samples with low proliferative BCs $(p=0.006)$, respectively. Pathologist 2 had no significant results for subgroup analysis and no other significant correlations were seen for high PTB. 
Fig. 2 Peripheral and intratumoral budding
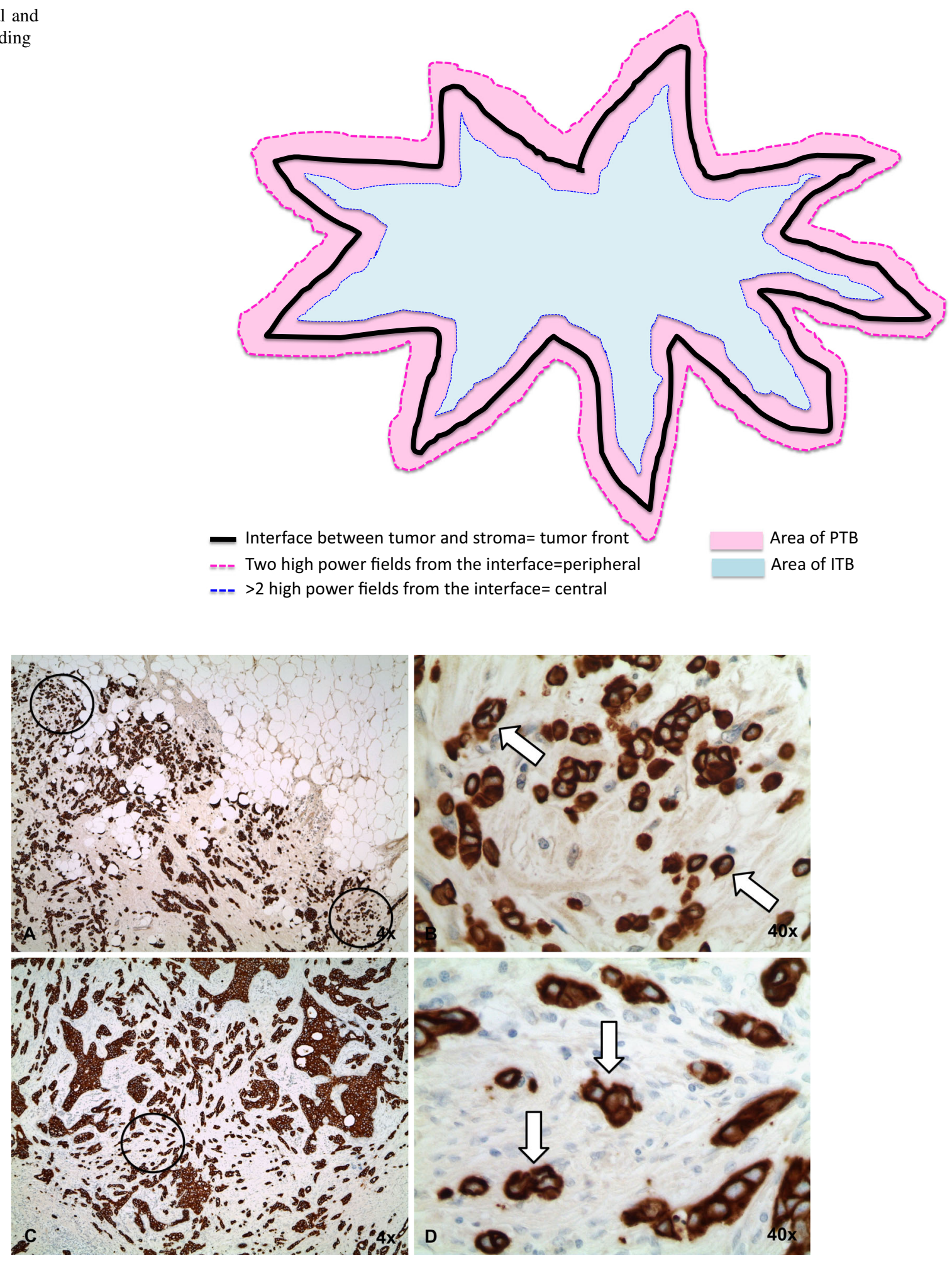

Fig. 3 NST breast cancer with high tumor budding. Overview $(\times 4)$ of a high PTB $($ a) and high ITB (c) breast cancer stained with anti-pancytokeratin antibody. $\mathbf{b}$ and $\mathbf{d}$ A high-power field $(\times 40)$ with more than four tumor buds 


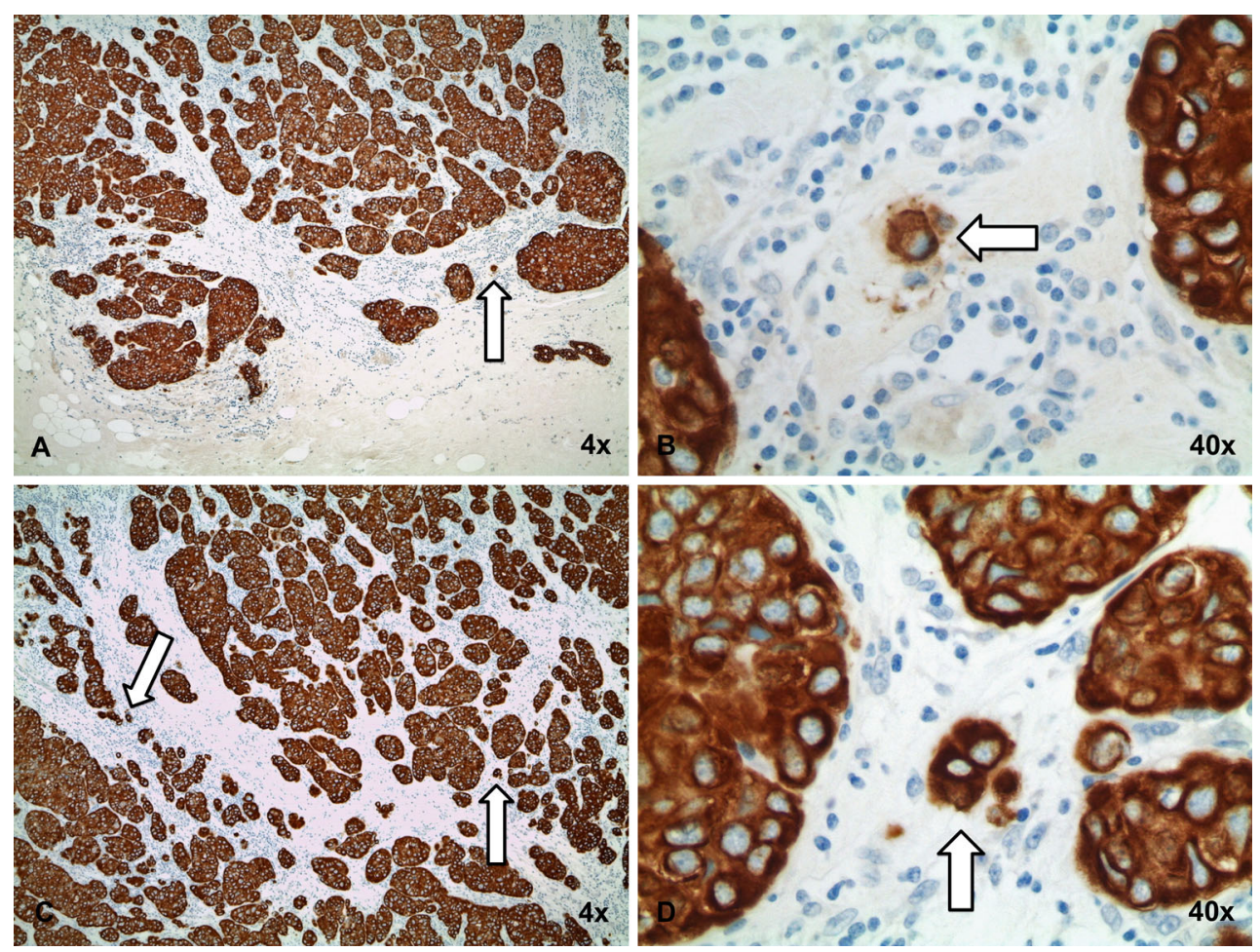

Fig. 4 NST breast cancer with low tumor budding. Overview $(\times 4)$ of a low PTB (a) and low ITB (c) breast cancer stained with anti-pancytokeratin antibody. $\mathbf{b}$ and $\mathbf{d}$ A high-power field $(\times 40)$ with less than four tumor buds

\section{Significant associations with ITB in resection specimens}

High ITB scoring results of Pathologist 1 showed a significant association with tumor grade $(p=0.036)$, ER positivity $(p=0.02)$ and lower proliferation $(p=0.002)$. The scoring results of Pathologist 2 demonstrated a significant association with negative Her2 status $(p=0.001)$ and perineural invasion $(p=0.039)$. The scoring results with significant associations are summarized in Table 3 and the subgroup analysis of PTB are summarized in Table 4.

\section{Significant associations of tumor budding in preoperative core biopsies}

Ninety-nine patients $(66.9 \%)$ with $\mathrm{CB}$ were identified from the 148 SRSs and evaluated for the presence of tumor budding. A significant positive correlation was found between budding in the CB and ITB $(r=0.28 ; p=0.0045)$ and PTB $(r=0.48 ; p<0.0001)$ of the SRS. In CB, tumor budding was significantly associated with $\mathrm{V}$ classification ( $p=0.0063$ ). All 35 patients with low-grade tumor budding $(<10$ buds/HPF) showed no venous invasion while $12 / 64(19 \%)$ patients with high-grade budding were diagnosed with venous invasion. No other significant associations were found with other pathological parameters (Table 4

\section{Discussion}

Tumor budding is best described as a histologic pattern associated with poor prognosis in early-stage colorectal adenocarcinoma and a predictor of nodal metastasis in $\mathrm{T} 1$ colorectal adenocarcinoma [18]. Recently, some of these associations were also found in esophageal carcinoma and pancreatic cancer $[19,20]$. Therefore, we investigated the significance of tumor budding and its relationship to known clinico-pathological features of BC such as nodal metastasis, tumor grade, and tumor size. In this retrospective study, we used a semiquantitative histologic scoring system to categorize 148 surgically resected, primary invasive NST BC's for the extent of both ITB and PTB. Additionally, 99 matching CBs were analyzed for tumor budding. We selected invasive ductal BC because they are the most prevalent histological subtypes [21] and represent a heterogeneous group with varying tumor features including immunphenotypical and clinical outcomes. Therefore, it would be of great clinical value to find additional prognostic or predictive tumor features for this group. 
Table 3 Summary of significant results and tumor budding

\begin{tabular}{|c|c|c|c|c|c|c|c|}
\hline \multirow[t]{2}{*}{ Features } & & \multicolumn{2}{|l|}{ PTB1 $n(\%)$} & \multirow[t]{2}{*}{$p$-value } & \multicolumn{2}{|l|}{ PTB2 $n(\%)$} & \multirow[t]{2}{*}{$p$-value } \\
\hline & & Low 30 (20.3) & High 118 (79.7) & & Low 17 (11.5) & High 131 (88.5) & \\
\hline \multirow[t]{2}{*}{$\mathrm{pN}$} & Positive & $9(11.2)$ & $71(88.8)$ & 0.003 & $5(6.2)$ & $75(93.8)$ & 0.03 \\
\hline & Negative & $21(30.9)$ & $47(69.1)$ & & $12(17.6)$ & $56(82.4)$ & \\
\hline \multirow[t]{2}{*}{ Lymphatic invasion } & Positive & $6(10.3)$ & $52(89.7)$ & 0.015 & $2(3.4)$ & $56(96.6)$ & 0.013 \\
\hline & Negative & $24(27)$ & $65(73)$ & & $15(16.9)$ & $74(83.1)$ & \\
\hline \multirow[t]{2}{*}{ Features } & & \multicolumn{2}{|l|}{ ITB1 $n(\%)$} & $p$-value & \multicolumn{2}{|l|}{ ITB2 $n(\%)$} & $p$-value \\
\hline & & Low 30 (20.9) & High 117 (79.1) & & Low 24 (16.2) & High 124 (83.8) & \\
\hline \multirow[t]{3}{*}{ Grading } & G1 & $3(16.7)$ & $15(83.3)$ & & $2(11.1)$ & $16(88.9)$ & \\
\hline & $G 2$ & $9(13.0)$ & $60(87.0)$ & 0.036 & $9(13.0)$ & $60(87.0)$ & 0.364 \\
\hline & G3 & $19(31.1)$ & $42(68.9)$ & & $13(21.3)$ & $48(78.7)$ & \\
\hline \multirow[t]{2}{*}{ ER } & Positive & $22(17.6)$ & $103(82.4)$ & 0.02 & $19(15.2)$ & $106(84.8)$ & 0.434 \\
\hline & Negative & $9(39.1)$ & $14(60.9)$ & & $5(21.7)$ & $18(78.3)$ & \\
\hline \multirow[t]{2}{*}{ Her2 } & Positive & $7(36.8)$ & $12(63.2)$ & 0.68 & $8(42.1)$ & $11(57.9)$ & 0.001 \\
\hline & Negative & $24(18.6)$ & $105(81.4)$ & & $16(12.4)$ & $113(87.6)$ & \\
\hline \multirow[t]{4}{*}{ Molecular subtypes } & Luminal A & $11(12.0)$ & $81(88.0)$ & & $11(12.0)$ & $81(88.0)$ & \\
\hline & Luminal B & $11(33.3)$ & $22(66.7)$ & 0.007 & $8(24.2)$ & $25(75.8)$ & 0.038 \\
\hline & Her2 & $1(33.3)$ & $2(66.7)$ & & $2(66.7)$ & $1(33.3)$ & \\
\hline & Triple negative & $8(40.0)$ & $12(60.0)$ & & $3(15.0)$ & $17(85.0)$ & \\
\hline \multirow[t]{2}{*}{ MIB1 } & High $(>15 \%)$ & $15(37.5)$ & $25(62.5)$ & 0.002 & $9(22.5)$ & $31(77.5)$ & 0.216 \\
\hline & Low $(\leq 15 \%)$ & $15(14.0)$ & $92(86.0)$ & & $15(14.0)$ & $92(86.0)$ & \\
\hline \multirow[t]{2}{*}{ Perineural invasion } & Positive & $1(5.3)$ & $18(94.7)$ & 0.7 & $0(0.0)$ & $19(100)$ & \\
\hline & Negative & $30(23.4)$ & $98(76.6)$ & & $24(18.8)$ & $104(81.2)$ & 0.039 \\
\hline
\end{tabular}

PTB1 peripheral tumor budding score from Pathologist 1

$P T B 2$ peripheral tumor budding score from Pathologist 2

ITB1 intratumoral budding score from Pathologist 1

ITB2 intratumoral budding score from Pathologist 2

Bold italic significant results

Table 4 Subgroup analysis with significant results

\begin{tabular}{|c|c|c|c|c|c|c|c|}
\hline \multirow[t]{2}{*}{ Features } & & \multicolumn{2}{|c|}{ PTB1 $n(\%)$} & \multirow[t]{2}{*}{$p$-value } & \multicolumn{2}{|c|}{ PTB2 $n(\%)$} & \multirow[t]{2}{*}{$p$-value } \\
\hline & & Low & High & & Low & High & \\
\hline \multirow[t]{2}{*}{ Only ER positive } & pN positive & $7(10.8)$ & $58(89.2)$ & & $5(7.7)$ & $60(92.3)$ & \\
\hline & pN negative & $18(30.0)$ & $42(70.0)$ & 0.007 & $10(16.7)$ & $50(83.3)$ & 0.123 \\
\hline \multirow[t]{2}{*}{ Only low MIB1 $(\leq 15 \%)$} & $\mathrm{pN}$ positive & $4(7.5)$ & $49(92.5)$ & 0.006 & $3(5.7)$ & $50(94.3)$ & 0.194 \\
\hline & $\mathrm{pN}$ negative & $15(27.8)$ & $39(72.2)$ & - & $7(13.0)$ & $47(87.0)$ & - \\
\hline
\end{tabular}

Bold italic significant results

During the course of our study, a paper was published by Liang et al. which demonstrated that high-grade budding in SRS was significantly associated with the presence of lymphatic invasion (LVI), larger tumor size, and worse clinical outcome [15]. We confirmed a significant association with LVI $(p=0.029)$ for PTB. Additionally, both pathologists were able to stratify BCs with positive lymph node metastases according to having a high PTB count ( $p=0.004$ and 0.03). Liang et al. reported that BC patients with high tumor bud scores have worse overall survival. Although long-term follow-up data were not available for the samples in this study, the association of tumor budding 
with positive lymph nodes suggests that tumor budding is a poor prognostic feature of BC. The limited number of patients and the fact that this is a retrospective study can be regarded as disadvantage. However, the endpint of this study was the lymph node status and not survival.

However, we did not find an association to tumor size or T-category as in the study by Liang et al. Certainly, the differences between the two studies could be related to the manner in which tumor buds were investigated. For our evaluation, we used IHC to better visualize hot-spots of single tumor cells or small tumor clusters and we defined tumor budding to be between 1 and 5 tumor cells and each pathologist scored $10 \mathrm{HPF}$ in SRS. In the Liang study, $\mathrm{H} \& \mathrm{E}$ was predominately used for scoring and tumor buds were defined as 1-4 tumor cells. Pathologists scored 5 "hot-spot" loci. In addition, we investigated ITB, which was not previously described.

Interestingly, in colon cancer, a cut-off of $>=10$ buds is proposed for high tumor budding [14]. In BC fewer tumor buds are required in SRS to stratify BC with adverse tumor features. The colon mucosa is much more involved in immune defense than breast tissue and for this reason may require a higher budding load to have adverse prognostic effects. Evaluating tumor budding in each tissue and specimen type separately will therefore be necessary to obtain meaningful prognostic data.

Epithelial-mesenchymal transition (EMT), a feature of metastatic cells, has previously been described in the context of tumor budding [22] and could be consistent with the observed association to both LVI and lymph node positivity but requires further analysis. A recent report also demonstrated that estrogen is involved in EMT in breast cancer cell lines with stem cell properties [23] and it was shown that estrogen is involved in disruption of tightjunction and increased cell motility [24]. We suspect that ER-positive tumors with high tumor budding may be undergoing a high degree of EMT and therefore have more metastatic potential. Further, the tumor center, which can have fibrosis, may represent a more hypoxic environment what might contribute to EMT transformation [25]. However, these hypotheses need further investigations.

In our study, we confirmed the presence of tumor budding in BC and its association to known poor prognostic indicators such as lymphatic invasion and positive lymph node status in univariate analysis in SRS. However, information on vascular invasion and lymph node status would be more relevant in the preoperative setting for therapeutic decisions. Therefore, we investigated tumor budding on matching preoperative $\mathrm{CBs}$ for the first time in BC. Since CB are randomly sampled and consist of smaller pieces of tissue, tumor buds cannot be classified as PTB or ITB and also require different scoring criteria. In $\mathrm{CBs,} \mathrm{we}$ identified a significant association of tumor buds with venous invasion, suggesting that $\mathrm{CBs}$ with high tumor budding might harbor a tumor cell population with an affinity for vascular invasion. The significance of this finding needs to befurther investigated. The lack of an association with LVI or positive nodal status as is observed in SRSs with high PTB could be explained if CB contain mostly ITB. Another explanation might be that the random capturing of tumor buds amidst a mix of PTB and ITB in CBs dilutes correlations of LVI and positive nodal status.

We demonstrated for the first time in $\mathrm{BC}$ that budding can be subdivided into ITB and PTB based on tumor localization, which may have disease implications. More research is needed to better understand the biological and clinical significance of ITB versus PTB in BC. Further, the concordance of scoring between the pathologists is better than the assessment of other tumor markers such as MIB1 [26]. Whether ITB could be used as an additional morphological feature to stratify ER-positive or low proliferative tumors $(\leq 15 \%)$ into a high and low risk category has to be investigated in a larger cohort. In addition, future studies should incorporate MIB1 analysis on whole tissue sections to bypass the reported caveat of obtaining significantly lower proliferation indices when using TMAs [27].

The evaluation of one tissue slide may not be ideal disadvantage, however, most tumor markers are evaluated on a single $\mathrm{CB}$ slide randomly selected from the tumor mass and with much less tumor content. Still however, implementation of tumor budding into the diagnostic setting requires further standardization to better define tumor buds and establish scoring parameters and cut-off criteria based on sensitivity and specificity. According to our data, tumor budding is a histological feature in BC with association to a more aggressive tumor phenotype and which may increase potential for metastasis. Our data demonstrate the utility of tumor budding in $\mathrm{BC}$ to potentially enhance prognostication in the clinic and warrants further investigation.

Acknowledgments We thank the tissue bank Bern and the Translational Research Unit (TRU) at the Institute of Pathology, University of Bern, Switzerland for ngTMA construction.

Conflict of interest The authors declare that they have no conflict of interest.

Funding This study was funded by the Claudia von Schilling Foundation, for Breast Cancer Research (Germany).

Open Access This article is distributed under the terms of the Creative Commons Attribution Noncommercial License which permits any noncommercial use, distribution, and reproduction in any medium, provided the original author(s) and the source are credited. 


\section{References}

1. Sorlie T, Perou CM, Tibshirani R, Aas T, Geisler S, Johnsen H, Hastie T, Eisen MB, van de Rijn M, Jeffrey SS, Thorsen T, Quist $\mathrm{H}$, Matese JC, Brown PO, Botstein D, Lonning PE, BorresenDale AL (2001) Gene expression patterns of breast carcinomas distinguish tumor subclasses with clinical implications. Proc Natl Acad Sci USA 98:10869-10874

2. Perou CM, Sorlie T, Eisen MB, van de Rijn M, Jeffrey SS, Rees CA, Pollack JR, Ross DT, Johnsen H, Akslen LA, Fluge O, Pergamenschikov A, Williams C, Zhu SX, Lonning PE, Borresen-Dale AL, Brown PO, Botstein D (2000) Molecular portraits of human breast tumours. Nature 406(6797):747-752

3. Polyak K, Hu M (2005) Do myoepithelial cells hold the key for breast tumor progression? J Mammary Gland Biol Neoplasia 10(3):231-247

4. Sims AH, Howell A, Howell SJ, Clarke RB (2007) Origins of breast cancer subtypes and therapeutic implications. Nat Clin Pract Oncol 4(9):516-525

5. van de Vijver MJ, He YD, van't Veer LJ, Dai H, Hart AA, Voskuil DW, Schreiber GJ, Peterse JL, Roberts C, Marton MJ, Parrish M, Atsma D, Witteveen A, Glas A, Delahaye L, van der Velde T, Bartelink H, Rodenhuis S, Rutgers ET, Friend SH, Bernards R (2001) A gene-expression signature as a predictor of survival in breast cancer. N Engl J Med 347:1999-2009

6. Gokmen-Polar Y, Badve S (2012) Breast cancer prognostic markers: where are we now? MLO Med Lab Obs 44(22):24-25

7. Schnitt SJ (2010) Classification and prognosis of invasive breast cancer: from morphology to molecular taxonomy. Mod Pathol 23(Suppl 2):S60-S64

8. Wessels LF, van Welsem T, Hart AA, van't Veer LJ, Reinders MJ, Nederlof PM (2002) Molecular classification of breast carcinomas by comparative genomic hybridization: a specific somatic genetic profile for BRCA1 tumors. Cancer Res 62:7110-7117

9. Lugli A, Karamitopoulou E, Zlobec I (2012) Tumour budding: a promising parameter in colorectal cancer. $\mathrm{Br} \mathrm{J}$ Cancer 106:1713-1717

10. McShane LM, Altman DG, Sauerbrei W, Taube SE, Gion M, Clark GM, Statistics Subcommittee of the NCI-EORTC Working Group on Cancer Diagnostics (2005) Reporting recommendations for tumor marker prognostic studies (REMARK). J Natl Cancer Inst 97:1180-1184

11. Zlobec I, Suter G, Perren A, Lugli A (2014) A next-generation tissue microarray (ngTMA) protocol for biomarker studies. J Vis Exp 23(91):e51893

12. Hammond ME, Hayes DF, Dowsett M, Allred DC, Hagerty KL, Badve S, Fitzgibbons PL, Francis G, Goldstein NS, Hayes M, Hicks DG, Lester S, Love R, Mangu PB, McShane L, Miller K, Osborne KC, Paik S, Perlmutter J, Rhodes A, Sasano H, Schwartz JN, Sweep FC, Taube S, Torlakovic EE, Valenstein P, Viale G, Visscher D, Wheeler T, Williams RB, Wittliff JL, Wolff AC (2010) American Society of Clinical Oncology/College Of American Pathologists guideline recommendations for immunohistochemical testing of estrogen and progesterone receptors in breast cancer. J Clin Oncol 16:2784-2795

13. Wolff AC, Hammond ME, Hicks DG, Dowsett M, McShane LM, Allison KH, Allred DC, Bartlett JM, Bilous M, Fitzgibbons P, Hanna W, Jenkins RB, Mangu PB, Paik S, Perez EA, Press MF, Spears PA, Vance GH, Viale G, Hayes DF (2013) Recommendations for human epidermal growth factor receptor 2 testing in breast cancer: American Society of Clinical Oncology/College of
American Pathologists clinical practice guideline update. J Clin Oncol 31(31):3997-4013

14. Karamitopoulou E, Zlobec I, Kölzer V, Kondi-Pafiti A, Patsouris ES, Gennatas K, Lugli A (2013) Proposal for a 10-high-powerfields scoring method for the assessment of tumor budding in colorectal cancer. Mod Pathol 26:295-301

15. Liang F, Cao W, Wang Y, Li L, Zhang G, Wang Z (2013) The prognostic value of tumor budding in invasive breast cancer. Pathol Res Pract 209(5):269-275

16. Zlobec I, Hädrich M, Dawson H, Koelzer VH, Borner M, Mallaev M, Schnüriger B, Inderbitzin D, Lugli A (2014) Intratumoral budding (ITB) in preoperative biopsies predicts the presence of lymph node and distant metastases in colon and rectal cancer patients. Br J Cancer 110(4):1008-1013

17. Landis JR, Koch CG (1977) The measurement of observer agreement for categorical data. Biometrics 33(1):159-174

18. Guzinska-Ustymowicz K (2005) The role of tumour budding at the front of invasion and recurrence of rectal carcinoma. Anticancer Res 25(2B):1269-1272

19. Niwa Y, Yamada S, Koike M, Kanda M, Fujii T, Nakayama G, Sugimoto H, Nomoto S, Fujiwara M, Kodera Y (2014) Epithelial to mesenchymal transition correlates with tumor budding and predicts prognosis in esophageal squamous cell carcinoma. J Surg Oncol 110(6):764-769

20. Karamitopoulou E, Zlobec I, Born D, Kondi-Pafiti A, Lykoudis P, Mellou A, Genatas K, Gloor B, Lugli A (2013) Tumour budding is a strong and independent prognostic factor in pancreatic cancer. Eur J Cancer 49(5):1032-1039

21. Lakhani SR, Ellis IO, Schnitt SJ, Tan PH, van de Vijver MJ (eds) (2012) WHO classification of tumours of the breast, 4the edn. IARC Press, Lyon

22. Zlobec I, Lugli A (2010) Epithelial mesenchymal transition and tumor budding in aggressive colorectal cancer: tumor budding as oncotarget. Oncotarget 1(7):651-661

23. Sun Y, Wang Y, Fan C, Gao P, Wang X, Wie G, Wie J (2014) Estrogen promotes stemness and invasiveness of ER-positive breast cancer cells through Gli1 activation. Mol Cancer 3(13):137. doi:10.1186/1476-4598-13-137

24. Jiménez-Salazar JE, Posadas-Rodriquez P, Lazzarini-Lechuga RC, Luna-Lopez A, Zentella-Dehesa A, Gomez-Quiroz LE, Königsberg M, Dominquez-Gomez G, Damian-Matsumura P (2014) Membrane-initiated estradiol signaling of epithelial-mesenchymal transition-associated mechanisms through regulation of tight junctions in human breast cancer cells. Horm Cancer 5(3):161-173

25. Chen H, Shen A, Zhang Y, Chen Y, Lin J, Lin W, Sferra T, Peng J (2014) Pien Tze Huang inhibits hypoxia-induced epithelialmesenchymal transition in human colon carcinoma cells through suppression of the HIF-1 pathway. Exp Ther Med $7(5): 1237-1242$

26. Varga Z, Diebold J, Dommann-Scherrer C, Frick H, Kaup D, Noske A, Obermann E, Oehlschlegel C, Padberg B, Rakozy C, Sancho Oliver S, Schobinger-Clement S, Schreiber-Facklam H, Singer G, Tapia C, Wagner U, Mastropasqua MG, Viale G, Lehr AH (2012) How reliable is Ki-67 immunohistochemistry in grade 2 breast carcinomas? A QA study of the Swiss working group of breast- and gynecopathologists. PLoS One 7(5):e37379

27. Knutsvik G, Stefansson IM, Aziz S, Arnes J, Eide J, Collett K, Akslen LA (2014) Evluation of Ki67 expression across distinct categories of breast cancer specimens: a population-based study of matched surgical specimens, core needle biopsies and tissue microarrays. PLoS One 9(11):e112121 